Artigo Original

\title{
Avaliação das condições de uso de esfigmomanômetros em serviços hospitalares*
}

\author{
Evaluation of the conditions of use of sphygmomanometers in hospital services
}

Evaluación de las condiciones de uso de esfigmomanómetros en servicios hospitalarios

\section{Talita de Souza Serafim¹, Gabriela de Andrade Toma', Josiane Lima de Gusmão², Flávia Cortez Colósimo ${ }^{3}$, Stael Silvana Bagno Eleutério da Silva ${ }^{3}$, Angela Maria Geraldo Pierin ${ }^{4}$}

\begin{abstract}
RESUMO
Objetivo: Avaliar as condições de uso dos esfigmomanômetros em hospitais públicos e privados. Métodos: Estudo descritivo de abordagem quantitativa realizado em quatro hospitais de grande porte do Estado de São Paulo, no período entre 2009 e 2010. Os manômetros aneroides foram testados contra manômetro de mercúrio calibrado. Foram considerados descalibrados quando as diferenças foram $\geq$ a $4 \mathrm{mmHg}$. Resultados: Foram avaliados 162 esfigmomanômetros, (78 de um hospital público e 84 de instituições filantrópicas e privada) e 98,1\% eram do tipo aneróide. Verificou-se que 56,2\% dos manômetros estavam descalibrados (48,6\% do hospital privado e 63,1\% dos hospitais públicos). Analisando-se as médias das diferenças negativas da descalibração, houve diferença significativa entre os manômetros do hospital privado e os dos hospitais públicos $(-6,14 \pm 2,66 \mathrm{mmHg} v s-8,97 \pm 6,74 \mathrm{mmHg}$, respectivamente, $\mathrm{p}<0,05)$. Observou-se ainda que em 70,2\% não era feita avaliação periódica; $26,7 \%$ tinham extensão de borracha envelhecida; $20,5 \%$ das válvulas apresentaram vazamento; e $27 \%$ dos manômetros não estavam com o ponteiro na marca zero. Conclusão A descalibração dos esfigmomanômetros aneróides foi expressiva e pode acarretar avaliação incorreta da pressão arterial. Descritores: Esfigmomanômetros; Determinação da pressão arterial/instrumentação; Falha de equipamento; Estudos de avaliação como assunto; Serviços hospitalares
\end{abstract}

\begin{abstract}
Objective: To evaluate the conditions of sphygmomanometers in use at public and private hospitals. Methods: A descriptive study using a quantitative approach, undertaken in four major hospitals in the State of São Paulo, in the period between 2009 and 2010. The aneroid manometers were tested against a calibrated mercury manometer. They were considered out of calibration when the differences were $\geq 4 \mathrm{mmHg}$. Results: We assessed 162 sphygmomanometers (78 in a public hospital and 84 from philanthropic and private institutions) and $98.1 \%$ were of the aneroid type. It was verified that $56.2 \%$ of the manometers were not calibrated ( $48.6 \%$ of private hospitals and $63.1 \%$ of public hospitals). Analyzing the mean differences of negative decalibration, there was a significant difference between the manometers of the private hospital and the public hospitals (-6.14 $\pm 2.66 \mathrm{mmHg}$ vs. $-8.97 \pm 6.74 \mathrm{mmHg}$, respectively, $\mathrm{p}<0.05)$. It was also observed that in $70.2 \%$ there was no periodic evaluation made, $26.7 \%$ had aged rubber extension, $20.5 \%$ presented leaking valves, and $27 \%$ of the manometers did not rest with the pointer on the zero mark. Conclusion: The decalibration of the aneroid sphygmomanometers was significant and may lead to incorrect evaluation of blood pressure.
\end{abstract}

Keywords: Sphygmomanometers; Blood pressure determination/instrumentation; Equipment failure; Evaluation studies as topic; Hospital services

\section{RESUMEN}

Objetivo: Evaluar las condiciones de uso de los esfigmomanómetros en hospitales públicos y privados. Métodos: Estudio descriptivo de abordaje cuantitativo realizado en cuatro hospitales de gran porte del Estado de Sao Paulo, en el período entre 2009 y 2010. Los manómetros aneroides fueron probados contra manómetro de mercurio calibrado. Se consideraron descalibrados cuando las diferencias fueron $\geq$ a 4 mmHg. Resultados: Fueron evaluados 162 esfigmomanómetros, (78 de un hospital público y 84 de instituciones filantrópicas y privadas) y el 98,1\% eran del tipo aneroide. Se verificó que el 56,2\% de los manómetros estaban descalibrados (48,6\% del hospital privado y 63,1\% de los hospitales públicos). Analizándose las medias de las diferencias negativas de la descalibración, hubo diferencia significativa entre los manómetros del hospital privado y los de los hospitales públicos $(-6,14 \pm 2,66 \mathrm{mmHg} v s-8,97 \pm 6,74 \mathrm{mmHg}$, respectivamente, $\mathrm{p}<0,05)$. Se observó aun que en el 70,2\% no se realiza la evaluación periódica; $26,7 \%$ tenían extensión de jebe envejecido; el 20,5\% de las válvulas presentaron derramamiento; y el 27\% de los manómetros no estaban con el puntero en la marca cero. Conclusión: La descalibración de los esfigmomanómetros aneroides fue expresiva y puede acarrear evaluación incorrecta de la presión arterial. Descriptores: Esfigmomanómetros; Determinación de la presión arterial/instrumentación; Falha de equipamento; Estudios de evaluación como asunto; Servicios hospitalarios

* Estudo realizado em quatro hospitais de grande porte do Estado de São Paulo, um hospital particular, duas Santas Casas e um hospital público.

${ }^{1}$ Enfermeira, graduada pela Escola de Enfermagem da Universidade de São Paulo - USP - São Paulo (SP), Brasil.

2 Professora Adjunto do Programa de Mestrado em Enfermagem da Universidade Guarulhos - Guarulhos (SP), Brasil.

${ }^{3}$ Pós-graduanda (Doutorado) do Programa de Enfermagem na Saúde do Adulto da Escola de Enfermagem, Universidade de São Paulo - USP, São Paulo (SP), Brasil.

${ }^{4}$ Professora Titular da Escola de Enfermagem, Universidade de São Paulo - USP - São Paulo (SP), Brasil. 


\section{INTRODUÇÃO}

Vários são os fatores que influenciam a medida da pressão arterial, destacando-se dentre eles, as condições dos esfigmomanômetros, sobretudo a calibração do manômetro. A medida da pressão arterial determina o diagnóstico e conduz o tratamento da hipertensão arterial, devendo ser realizada de forma correta para evitar diagnóstico errôneo. Se, por um lado, o diagnóstico de normotensão em indivíduo hipertenso irá privá-lo de tratamento adequado, por outro, o diagnóstico de hipertensão em normotenso irá submetê-lo a tratamento desnecessário. Desta forma, esfigmomanômetros precisos e funcionando adequadamente, são essenciais para se obter uma medida fiel da pressão arterial. Outro fator a ser destacado é o aspecto organizacional da instituição de saúde que pode influenciar diretamente as condições dos equipamentos, uma vez que instituições públicas ou filantrópicas que dependem de verba do Sistema Único de Saúde ou doações podem ser menos favorecidas e ter maior dificuldade na aquisição de equipamentos de qualidade e na realização de sua manutenção. Por outro lado, instituições privadas estariam menos expostas a essas dificuldades.

$\mathrm{Na}$ prática clínica, em nosso meio, a medida indireta da pressão arterial era realizada, geralmente, com uso de esfigmomanômetros de coluna de mercúrio e aneróide. Mas, o Ministério do Trabalho proibiu o uso do mercúrio em ambiente hospitalar (NR 15 125.001-9/I4) ${ }^{(1)}$ limitando, dessa forma, emprego dos esfigmomanômetros de coluna de mercúrio. A partir de então, em muitas instituições, os esfigmomanômetros aneroides passaram a ser o único tipo de aparelho usado para a medida da pressão arterial.

Entretanto, estudos nacionais e internacionais vêm mostrando condições de calibração inadequadas dos manômetros aneroides ${ }^{(2-6)}$. Dada à estrutura e manutenção simples do esfigmomanômetro de coluna de mercúrio, raramente se observa a perda de sua calibração. Tal calibração pode ser garantida tão somente, observando o nível do menisco de mercúrio que deve estar no ponto zero da escala de medida do aparelho, o que não acontece com o aneroide, pois o fato do ponteiro permanecer no ponto zero não significa que está calibrado. O esfigmomanômetro de coluna de mercúrio foi considerado o padrão ouro na medida da pressão arterial por mais de um século. Sua substituição por novos aparelhos vem sendo recomendada, tendo em vista a toxicidade do mercúrio, os erros humanos relacionados ao método auscultatório, a variabilidade da pressão arterial e a tendência do aumento da pressão arterial na presença do profissional de saúde $^{(7)}$. Entretanto, exposições acidentais ao mercúrio dos esfigmomanômetros são raras ${ }^{(8)}$.

Recomenda-se que os esfigmomanômetros aneroides ou de coluna de mercúrio sejam avaliados periodicamente, pelo menos, a cada 6 meses. Além, de que o Instituto $\mathrm{Na-}$ cional de Pesos e Medidas estabeleceu normatização, prevendo que todos os manômetros aneroides tenham selo de garantia emitido por este órgão para serem comercializados e, uma vez em uso, devem ser periodicamente testados ${ }^{(9)}$.

Face aos aspectos destacados e considerando que, em nosso meio, os estudos ${ }^{(2-4)}$ que avaliaram as condições dos esfigmomanômetros mostraram a precariedade de seu estado de conservação, bem como elevados índices de descalibração, sobretudo, em instituições públicas, o presente estudo teve como objetivo avaliar as condições de uso dos aparelhos de medida da pressão arterial em hospitais públicos e privados.

\section{MÉTODOS}

Realizou-se estudo descritivo, com análise quantitativa, no qual foram escolhidos de forma aleatória, no período entre 2009-2010, quatro hospitais de grande porte do Estado de São Paulo, um hospital particular, duas Santas Casas e um hospital público. Para efeito de análise, as duas Santas Casas e o hospital público foram reunidos em "Hospitais Públicos". Foi solicitada autorização para a realização do estudo junto às instituições, bem como a assinatura do Termo de Consentimento Livre e Esclarecido e, somente, após esta etapa, foram aplicadas as questões do formulário para os enfermeiros responsáveis pelas unidades, seguida da avaliação dos esfigmomanômetros. Foram avaliados todos os esfigmomanômetros aneroides e de coluna de mercúrio em uso nas diferentes unidades dos hospitais. A coleta de dados foi realizada por alunos de graduação e pós-graduação da Escola de Enfermagem da Universidade de São Paulo (USP).

O instrumento utilizado para a coleta de dados constou de um formulário com questões fechadas e abertas, que foi preenchido pelos pesquisadores no momento da avaliação dos aparelhos. Os aparelhos foram avaliados nos seguintes aspectos: estado de conservação do manguito; integridade das extensões de borracha; integridade da pera de borracha; funcionalidade da válvula e presença de vazamento; e calibração dos manômetros.

Para avaliar a calibração do manômetro aneroide, foi feito um teste contra um de coluna de mercúrio, utilizando conector em forma de $\mathrm{Y}$, de acordo com o seguinte procedimento:

1) conectar o tubo de borracha do manômetro aneroide a ser testado em uma extremidade do "Y", o tubo de borracha do manômetro de coluna de mercúrio na outra extremidade e a pera de borracha para inflar o sistema na porção inferior do "Y";

2) inflar o sistema lentamente até $300 \mathrm{~mm} \mathrm{Hg}$;

3) abrir lentamente a válvula da pera de borracha, para redução da pressão;

4) verificar a correspondência de valores entre os dois manômetros de $10 \mathrm{em} 10 \mathrm{~mm} \mathrm{Hg}$, durante a inflação e deflação do sistema; e 
5) identificar a magnitude da diferença da correspondência de valores em $\mathrm{mmHg}$ em cada um dos níveis testados.

Os manômetros aneroides foram considerados descalibrados, quando as diferenças foram maiores ou iguais a 4 $\mathrm{mm} \mathrm{Hg}$ entre as duas escalas, em qualquer um dos pontos avaliados. Foram consideradas diferenças aceitáveis até 3 $\mathrm{mmHg}$ que corresponderam a $1 \%$ da escala do manômetro, conforme a Portaria do INMETRO no 153/2005.

O projeto de pesquisa foi aprovado por Comitê de Ética em Pesquisa da Escola de Enfermagem da USP (Processo no 644/2007CEP-EEUSP).

Os dados são apresentados na forma de tabelas e figura com valores absolutos e percentuais. Para os aparelhos descalibrados, foram computadas as diferenças com o aparelho-padrão (coluna de mercúrio) e expostas na forma de média \pm desvio-padrão. $\mathrm{Na}$ comparação entre os diferentes locais, público e privado, os aparelhos das entidades públicas foram reunidos $(\mathrm{n}=84)$ e avaliados contra os do hospital privado $(\mathrm{n}=78)$. Considerou-se significante valor de $\mathrm{p}<0,05$. Foi usado o teste Mann-Withney para comparar as diferenças entre as médias dos manômetros.

\section{RESULTADOS}

Foram avaliados 162 esfigmomanômetros e a quase totalidade $(98,1 \%)$ era do tipo aneroides, sendo apenas três aparelhos de mercúrio. Quanto às condições dos manguitos avaliados, $13 \%$ apresentavam sujidade ou presença de sangue e, em uma minoria, foram identificados rasgos $(2,5 \%)$. Cerca de um terço das peras e das extensões estava com a borracha envelhecida e, pouco menos, das válvulas de fechamento com vazamento (Tabela 1 ).

Tabela 1 - Características dos esfigmomanômetros de quatro hospitais de grande porte. São Paulo, 2009/2010

\begin{tabular}{|c|c|c|c|}
\hline \multicolumn{2}{|l|}{ Variáveis } & $\mathrm{n}$ & $(\%)$ \\
\hline \multicolumn{4}{|l|}{ Tipo } \\
\hline \multicolumn{2}{|l|}{ Aneroide } & 159 & 98,1 \\
\hline \multicolumn{2}{|c|}{ Aparelho de Mercúrio } & 3 & 1,9 \\
\hline \multicolumn{4}{|c|}{ Condições } \\
\hline \multirow[t]{3}{*}{ Manguito: } & Limpo & 138 & 85,7 \\
\hline & Sujidade/ Sangue & 21 & 13 \\
\hline & Rasgado & 4 & 2,5 \\
\hline \multirow[t]{2}{*}{ Pêra: } & Íntegra & 110 & 69,6 \\
\hline & Borracha envelhecida & 46 & 29,1 \\
\hline \multicolumn{4}{|c|}{ Extensão de borracha: } \\
\hline \multicolumn{2}{|c|}{ Íntegra } & 118 & 73,3 \\
\hline \multicolumn{2}{|c|}{ Envelhecida } & 43 & 26,7 \\
\hline \multicolumn{2}{|c|}{ Sujidade / Sangue } & 3 & 1,9 \\
\hline \multicolumn{4}{|c|}{ Válvula de fechamento: } \\
\hline \multicolumn{2}{|c|}{ Boas condições de fechamento } & 124 & 77 \\
\hline \multicolumn{2}{|c|}{ Vazamento } & 33 & 20,5 \\
\hline
\end{tabular}

A avaliação dos manguitos revelou que, na maioria expressiva $(81,4 \%)$, predominou o tipo de fechamento com as hastes de metal e parte delas não estava íntegra ou estava incompleta (15,3\%). Nos manguitos que eram de velcro, $20 \%$ apresentaram comprometimento de aderência. Quanto às dimensões dos manguitos, verificou-se que boa parte dos aparelhos avaliados não possuía manguito maior para medida da pressão em obesos $(88,9 \%)$ e menores para pessoas com braços finos $(76,4 \%)$. Mas, praticamente metade dos informantes referiu usar o manguito adequado para medida da pressão arterial em pessoas obesas, e 38,3\% utilizavam o manguito normal. Quanto à medida em pessoas com braços finos, foi expressiva $(56,2 \%)$ a referência de uso de manguito de tamanho padrão (Tabela 2).

Tabela 2 - Características dos manguitos dos esfigmomanômetros de quatro hospitais de grande porte. São Paulo, 2009-2010

\begin{tabular}{|c|c|c|}
\hline Variáveis & $\mathbf{n}$ & $(\%)$ \\
\hline \multicolumn{3}{|c|}{ Tipo de fechamento do manguito } \\
\hline Velcro & 30 & 18,6 \\
\hline Metal & 131 & 81,4 \\
\hline \multicolumn{3}{|l|}{ Condições } \\
\hline \multicolumn{3}{|l|}{ Metal: } \\
\hline Íntegras & 111 & 84,7 \\
\hline Amassadas/entortadas & 16 & 12,2 \\
\hline Incompletas & 4 & 3,1 \\
\hline \multicolumn{3}{|l|}{ Velcro: } \\
\hline Limpo & 28 & 93,3 \\
\hline Sujidade/sangue & 2 & 6,7 \\
\hline Aderente & 24 & 80 \\
\hline \multicolumn{3}{|l|}{ Possui manguito para obeso } \\
\hline Sim & 18 & 11,1 \\
\hline Não & 144 & 88,9 \\
\hline \multicolumn{3}{|c|}{ Quando atende obeso, o que faz? } \\
\hline Não realiza a medida & 2 & 1,2 \\
\hline Usa manguito padrão & 62 & 38,3 \\
\hline Mede no antebraço & 10 & 6,2 \\
\hline Usa manguito adequado & 82 & 50,6 \\
\hline Outro & 6 & 3,7 \\
\hline \multicolumn{3}{|c|}{ Possui manguito para braço fino } \\
\hline Sim & 38 & 23,6 \\
\hline Não & 123 & 76,4 \\
\hline \multicolumn{3}{|c|}{ Quando atende indivíduos com braço fino, o que faz? } \\
\hline Não realiza a medida & 9 & 5,6 \\
\hline Usa manguito padrão & 91 & 56,2 \\
\hline Usa manguito adequado & 57 & 35,2 \\
\hline Outro & 5 & 3,1 \\
\hline
\end{tabular}


A inspeção dos manômetros indicou que $27 \%$ não estavam com o ponteiro na marca zero, $15,1 \%$ com o ponteiro acima e $11,9 \%$ com o ponteiro abaixo da marca zero. Quanto às condições dos manômetros, apesar da maioria apresentar a parte externa em boas condições $(89,7 \%), 5,8 \%$ estavam com o vidro rachado. O selo de garantia obrigatória do INMETRO não estava presente em mais de um quarto dos aparelhos $(27,8 \%)$ e, em apenas cerca de um terço dos aparelhos, a calibração era testada regularmente; no entanto, a maioria dos entrevistados (76,6\%) não soube responder com que frequência era realizada. Quase a totalidade dos aparelhos não possuía data de última avaliação $(96,3 \%)$ e apenas $3,1 \%$ foram avaliados nos últimos três meses (Tabela 3).

Tabela 3 - Avaliação das condições de calibração dos manômetros de quatro hospitais de grande porte. São Paulo, 2009-2010

\begin{tabular}{lll}
\hline Variáveis & $\mathbf{n}$ & $\mathbf{( \% )}$ \\
\hline Ponteiro do manômetro aneroide & & \\
Na marca zero & 116 & 73 \\
Acima da marca zero & 24 & 15,1 \\
Abaixo da marca zero & 19 & 11,9
\end{tabular}

\section{Condições}

Vidro rachado

$9 \quad 5,8$

Parte externa em boas condições

$139 \quad 89,7$

Selo do INMETRO

$\begin{array}{lll}\text { Sim } & 117 & 72,2 \\ \text { Não } & 45 & 27,8\end{array}$

Calibração testada regularmente $\operatorname{Sim}$

Qual a frequência?

$$
\begin{aligned}
& 3 \text { meses } \\
& 6 \text { meses } \\
& \text { Não sabe } \\
& \text { Outro }
\end{aligned}
$$

Não

Data da última avaliação

\begin{tabular}{ccc} 
Não Sem informação? & 156 & 96,3 \\
Últimos 3 meses & 5 & 3,1 \\
\hline
\end{tabular}

A avaliação da calibração dos manômetros aneroides testados contra o manômetro de coluna de mercúrio revelou que do total dos aparelhos, $56,2 \%$ estavam descalibrados, sendo $48,7 \%$ do hospital privado e $63,1 \%$ dos hospitais públicos (Figura 1).

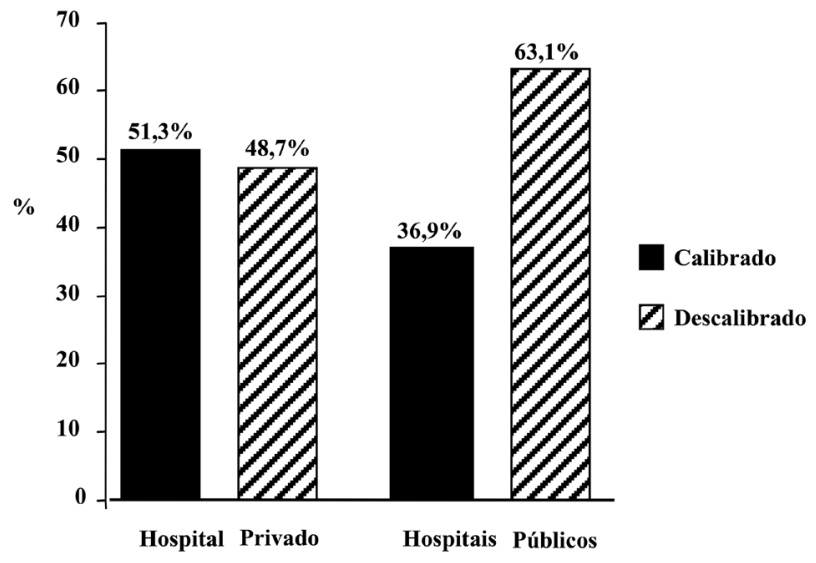

Figura 1 - Calibração dos manômetros entre instituições privada e públicas. São Paulo, 2009-2010.

A avaliação entre os manômetros aneroides testados contra o manômetro de coluna de mercúrio que foi o padrão usado, revelou que nos aparelhos dos hospitais privado e públicos, respectivamente, as diferenças foram: na faixa de 4 a $9 \mathrm{mmHg}, 41 \%$ e $45,2 \%$; de 10 a 14 $\mathrm{mmHg}, 3,8 \%$ e $9,5 \%$; e acima de $14 \mathrm{mmHg}, 3,8 \%$ e $8,3 \%$. Avaliando-se a magnitude das médias das diferenças, observa-se pelos dados da Tabela 4 que houve diferenças positivas e negativas. Ao comparar essas diferenças entre as instituições públicas e a privada, houve diferença estatisticamente significante para os valores negativos $(-8,97 \pm 6,74$ vs $-6,14 \pm 2,66 \mathrm{~mm} \mathrm{Hg})$, ou seja, os manômetros aneroides indicaram valores acima do apontado no manômetro de coluna de mercúrio; o mesmo ocorreu com a diferença total $(7,72 \pm 5,81$ vs $6,32 \pm 4,14)$.

\begin{tabular}{|c|c|c|c|c|}
\hline Variáveis & $\begin{array}{c}\text { Média } \pm \text { Desvio } \\
\text { Padrão } \\
\text { (mmHg) }\end{array}$ & $\begin{array}{c}\text { Valor } \\
\text { mínimo } \\
(\mathrm{mmHg})\end{array}$ & $\begin{array}{c}\begin{array}{c}\text { Valor } \\
\text { máximo } \\
(\mathrm{mmHg})\end{array} \\
\end{array}$ & $\begin{array}{l}\text { Valor } \\
\text { de p }\end{array}$ \\
\hline \multicolumn{5}{|l|}{$\begin{array}{l}\text { Hospital } \\
\text { privado }\end{array}$} \\
\hline $\begin{array}{l}\text { Diferença } \\
\text { para menos }\end{array}$ & $-6,14 \pm 2,66$ & -10 & -4 & $<0,001$ \\
\hline $\begin{array}{l}\text { Diferença } \\
\text { para mais }\end{array}$ & $6,38 \pm 4,44$ & 4 & 30 & $=0,097$ \\
\hline $\begin{array}{l}\text { Diferença } \\
\text { total }\end{array}$ & $6,32 \pm 4,14$ & 4 & 30 & $<0,001$ \\
\hline \multicolumn{5}{|l|}{$\begin{array}{l}\text { Hospitais } \\
\text { públicos }\end{array}$} \\
\hline $\begin{array}{l}\text { Diferença } \\
\text { para menos }\end{array}$ & $-8,97 \pm 6,74$ & -38 & -4 & \\
\hline $\begin{array}{l}\text { Diferença } \\
\text { para mais }\end{array}$ & $5,60 \pm 2,58$ & 4 & 22 & \\
\hline $\begin{array}{l}\text { Diferença } \\
\text { total }\end{array}$ & $7,72 \pm 5,81$ & 4 & 38 & \\
\hline
\end{tabular}

Tabela 4 - Diferenças da calibração dos manômetros aneroides entre as instituições hospitalares privada e públicas. São Paulo, 2009-2010 


\section{DISCUSSÃO}

O dado de que pouco mais da metade $(56,2 \%)$ dos manômetros aneroides testados apresentou-se descalibrado é um achado expressivo e preocupante, já que se tratava de aparelhos em uso nas instituições hospitalares onde o estudo foi realizado. A descalibração encontrada assemelha-se ao achado do primeiro estudo desse tipo realizado em nosso meio. Em 1998, mostrou-se que de um total de 524 aparelhos avaliados, 204 esfigmomanômetros aneróides e 320 esfigmomanômetros de coluna de mercúrio, 58\% dos aneroides estavam descalibrados contra $21 \%$ dos de coluna de mercúrio ${ }^{(2)}$. Com base nesse estudo, que o INMETRO passou a investigar o assunto, culminando na legislação específica para o uso dos esfigmomanômetros aneroides.

O presente estudo mostrou que, a quase totalidade dos esfigmomanômetros utilizados pelas instituições $(98,1 \%)$ são aneroides. No entanto, sabe-se que esses equipamentos são facilmente danificados e descalibram-se com facilidade com os choques cotidianos e quedas. Infelizmente, a maior descalibração dos manômetros aneroides em comparação com os aparelhos de coluna de mercúrio ainda é um fato evidenciado em vários estudos ${ }^{(3-6,10)}$. Estudos nacionais anteriores à recomendação da eliminação do mercúrio realizados em nosso meio, já mostravam a preferência pelos equipamentos aneroides, com taxas que variavam entre $59,6 \%{ }^{(11)}$ e $67,8 \%{ }^{(12)}$.

Muitos países, sobretudo na Europa, não utilizam mais esfigmomanômetros de mercúrio, porém eles foram substituídos por aparelhos automáticos e não aneroides, fato muito pouco frequente em nosso meio. A Organização Mundial de Saúde ${ }^{(13)}$, recentemente, publicou um manual com as devidas recomendações para a substituição dos termômetros e esfigmomanômetros de mercúrio, considerando, em especial, os danos da toxicidade do mercúrio ao meio ambiente. Nesse documento, é ressaltada a importância das adequadas condições dos esfigmomanômetros a serem usados, sobretudo a calibração. Destaca-se que, frequentemente, na aquisição dos equipamentos não se considera prioritária sua qualidade pela existência de uma relação inversa entre qualidade e custo, ocasionando a compra de esfigmomanômetros aneroides de qualidade insatisfatória facilitando ainda mais a descalibração do sistema. Essa constatação talvez possa explicar a diferença na calibração dos equipamentos entre as instituições públicas e privada avaliadas. Verificou-se que os esfigmomanômetros da instituição privada estavam em melhores condições de uso, ou seja, menos descalibrados (48,7\%) que os das públicas (63,1\%). As instituições privadas não dependem de verba pública e talvez tenham condições de adquirir equipamentos de boa qualidade. Vale ressaltar, no entanto, que apesar da diferença, 48,7\% de descalibração ainda é um taxa muito aquém do desejado. É importante lembrar que avaliações frequentes desses esfigmomanômetros, pelo menos, a cada seis meses, são necessárias. $\mathrm{O}$ teste do manômetro aneroide contra o de mercúrio devidamente calibrado pode ser facilmente realizado, utilizando-se um conector em forma de "Y". Nas duas extremidades superiores, são adaptados os dois manômetros e na porção inferior do conector, uma extensão de borracha com a pera para inflar. Dessa forma, é possível realizar a comparação dos valores dos dois manômetros na inflação e deflação do sistema. Essa é uma forma "caseira" de avaliação, pois a ideal é realizada por aparelho eletrônico de geração de pressão, cuja precisão é mais fidedigna.

O uso de aparelhos automáticos ou semiautomáticos para a medida da pressão arterial é uma realidade cada vez mais crescente na prática clínica e em investigações, pois, minimizam erros relacionados ao observador que realiza a medida, tais como: preferência por dígitos terminais "zero" e "cinco", ausculta inadequada dos sons que determinam a pressão sistólica e a diastólica, interação com o paciente, ocasionando o efeito do avental branco, dentre outros. Entretanto, os aparelhos devidamente adequados para uso têm custo mais elevado do que muitos aparelhos aneroides. Esses aparelhos só estão aptos para o uso se tiverem sido submetidos e aprovados por estudos de validação, de acordo com protocolos como os preconizados por entidades como a British Hypertension Society ${ }^{(14)}$, Association for the Advancement of Medical Instrumentation ${ }^{(15)} \mathrm{e}$ European Society of Hypertension ${ }^{(16)}$. Uma forma confiável para se identificar se o aparelho foi validado ou não é consultar os sites da dabl Educational (http:/ / www.dableducational.org/sphygmomanometers/devices_2_sbpm. html) e da British Hypertension Society (http:/ / www.bhsoc. org/bp_monitors/automatic.stm) que, além de fornecerem dados sobre a validação do equipamento, incluem também o preço. Ressalta-se que, mesmo aprovados, os aparelhos automáticos e semiautomáticos de medida da pressão arterial necessitam de revisão periódica, geralmente, realizada pelo fabricante ou autorizado.

Outro achado que merece atenção foi que a magnitude da descalibração dos manômetros de hospital público, avaliada pelas médias das diferenças, foi maior que a dos manômetros pertencentes ao hospital privado, o que pode comprometer ainda mais a fidedignidade da medida da pressão arterial. Nos hospitais públicos, encontrou-se manômetro com diferença negativa de até $38 \mathrm{mmHg}$. Os manômetros com diferenças negativas podem deixar de diagnosticar a hipertensão arterial e privar as pessoas dos benefícios do tratamento anti-hipertensivo, sujeitando-os a possíveis lesões de órgãos alvo. Estudo realizado em nosso meio há cerca de uma década também encontrou predomínio de diferenças negativas ${ }^{(17)}$. Em outra investigação que realizou simulação da medida da pressão arterial com aparelhos descalibrados, foi demonstrado que, 
após três consultas, os esfigmomanômetros descalibrados deixariam de diagnosticar $20 \%$ de hipertensão sistólica e 28\% de diastólica, sendo responsáveis pelo diagnóstico errôneo de hipertensão em 15\% das pressões sistólicas e $31 \%$ das pressões diastólicas ${ }^{(18)}$.

Acrescenta-se ainda, que a inadequada calibração dos manômetros aneroides encontrada relaciona-se ao fato de que $70 \%$ dos entrevistados referiram que não eram avaliados com frequência, e os que responderam sim, a maioria não sabia informar com qual frequência a avaliação era realizada, citando que quando observavam o ponteiro fora do ponto zero, é que consideravam o aparelho descalibrado. Dados semelhantes foram encontrados em um inquérito realizado ${ }^{(12)}$ com médicos, no qual apenas um terço dos entrevistados referiu verificar a calibração dos esfigmomanômetros com periodicidade inferior a um ano. Por outro lado, estudo semelhante realizado alguns anos mais tarde, mostrou dados bastante diferentes, com $72,9 \%$ dos médicos referiu verificar a calibração do aparelho em períodos inferiores a um ano ${ }^{(11)}$.

O presente estudo demonstrou também que a maioria dos locais visitados não possuía manguito de tamanho adequado para braços maiores como o de pessoas obesas e braços mais finos ( $88,9 \%$ e $76,4 \%$, respectivamente) e quando se encontravam nestas situações, na maioria das vezes, usava-se o manguito de tamanho padrão, o que pode acarretar em resultado errôneo da pressão arterial, hiperestimando ou subestimando o valor da pressão arterial. Investigação mostrou que o manguito correto diminuiu a hiperestimação dos registros diastólicos ${ }^{(19)}$, e forneceu valores de pressão mais próximos daqueles obtidos por equipamento fotopletismográfico ${ }^{(20)}$, considerado padrão ouro na medida indireta da pressão arterial.

A relação adequada entre o tamanho do braço e a largura da bolsa de borracha do manguito é que esta deve corresponder a $40 \%$ da circunferência do braço avaliada entre o ponto médio das proeminências ósseas do acrômio e olecrano ${ }^{(9)}$. Estudo realizado com profissionais da saúde sobre o conhecimento sobre a medida da pressão arterial, em específico, a dimensão do manguito, revelou desconhecimento dos profissionais quanto ao uso das diferentes dimensões do manguito ${ }^{(21)}$.

Além de se garantir que o manômetro esteja calibrado, a condição das demais partes do esfigmomanômetro também merece atenção. As más condições da pera e a extensão de borracha podem contribuir para avaliação errônea da pressão arterial. Apesar de grande parte dos manguitos estar limpa, com extensões e peras íntegras e sem vazamentos, é importante salientar que problemas de vazamento na válvula tornam difícil a inflação da bolsa de borracha e na deflação haverá dificuldade de controle da velocidade, podendo ocasionar erros na leitura, com falsa diminuição da pressão sistólica e elevação da pressão diastólica.

Os dados do presente estudo corroboram achados importantes e preocupantes que podem justificar ações em instituições de saúde públicas e privadas, que tenham por objetivo melhorar a qualidade e confiabilidade dos esfigmomanômetros por elas utilizados, por meio da verificação periódica dos equipamentos e substituição daqueles em condições inadequados para uso.

\section{CONCLUSÃO}

Observou-se que os esfigmomanômetros avaliados apresentaram descalibração expressiva tanto nos hospitais públicos, como no privado, embora esse achado tenha sido pior nas instituições públicas. Além da descalibração, faltavam manguitos adequados para medida em pessoas obesas e adultos magros, e os equipamentos não passavam por verificação regular da calibração. Esse conjunto de deficiências pode levar a uma avaliação incorreta dos níveis de pressão arterial. A importância dos profissionais da saúde, especialmente, o enfermeiro, de orientar sua equipe e ter o conhecimento de saber identificar a necessidade de reavaliar a calibração do aparelho de pressão arterial é de extrema importância para se garantir a obtenção de uma avaliação correta da pressão arterial, garantindo, assim, o seguimento de uma terapêutica correta.

A medida da pressão arterial é o procedimento mais realizado na área da saúde e deve ser feita em qualquer avaliação, independente da especialidade e em todas as faixas etárias, sendo inclusive obrigatória para crianças, a partir de três anos. Dessa forma, garantir adequadas condições do equipamento para a medida da pressão arterial é responsabilidade daqueles que a realizam. Em nosso meio, os profissionais de enfermagem detêm grande parte dessa responsabilidade que se inicia desde a apreciação e indicação do melhor equipamento, visando a garantir valores de pressão fidedignos.

Uma vez em uso, a avaliação periódica dos esfigmomanômetros deve ser estabelecida por uma rotina, conforme preconizado pelas Diretrizes Brasileiras de Hipertensão Arterial, de modo que seja possível identificar o mais precocemente possível qualquer alteração que prejudique a obtenção de valores corretos de pressão arterial. 


\section{REFERÊNCIAS}

1. Brasil. Ministério do Trabalho e Emprego. NR 15 Atividades e operações insalubres [Internet] [citado 2011 Set 10]. Disponível em: http://portal.mte.gov.br/data/files/ FF8080812DF396CA012E0017BB3208E8/NR-15\%20 (atualizada_2011).pdf.

2. Mion D, Pierin AM. How accurate are sphygmomanometers? J Hum Hypertens. 1998; 12(4):245-8.

3. Nobre F, Coelho EB, Dallora ME, Figueiredo PA, Ferreira AB, Rosa MA. Assessment of sphygmomanometers: a proposal for excellence in blood pressure measurement . Arq Bras Cardiol. 2009; 93(2):39-41.

4. Palota L, Cordella MP, Oliveira SM, Cesarino CB. [Evaluating the manometers calibration accuracy and conditions of the aneroid sphygmomanometers: a continuing coordinators program nursing education at Hospital de Base]. Arq Ciênc Saúde. 2004; 11(2): 2-6. Portuguese.

5. Waugh JJ, Gupta M, Rushbrook J, Halligan A, Shennan AH. Hidden errors of aneroid sphygmomanometers. Blood Press Monit. 2002;7(6):309-12.

6. Parati G, Faini A, Castiglioni P. Accuracy of blood pressure measurement: sphygmomanometer calibration and beyond. J Hypertens. 2006; 24(10):1915-8.

7. Pickering TG, Hall JE, Appel LJ, Falkner BE, Graves $\mathrm{G}$, Hill MN, et al. Recommendations for blood pressure measurement in humans and experimental animals: Part 1: blood pressure measurement in humans: a statement for professionals from the Subcommittee of Professional and Public Education of the American Heart Association Council on High Blood Pressure Research. Hypertension. 2005; 45(1):142-61.

8. Jones DW, Frohlich ED, Grim CM, Grim CE, Taubert KA. Mercury sphygmomanometers should not be abandoned: An advisory statement from the Council for High Blood Pressure Research, American Heart Association. Hypertension, 2001; 37(2):185-6.

9. Sociedade Brasileira de Cardiologia; Sociedade Brasileira de Hipertensão; Sociedade Brasileira de Nefrologia. VI Diretrizes Brasileiras de Hipertensão. Arq Bras Cardiol. 2010; 95(1 supl.1):1-51.

10. Buchanan S, Orris P, Karliner J. Alternatives to the mercury sphygmomanometer. J Public Health Policy. 2011;32(1):107-20.

11. Mion Júnior D, Silva GV, Gusmão JL, Machado CA,
Amodeo C, Nobre F et al . Do Brazilian physicians follow the Brazilian guidelines for hypertension?]. Arq Bras Cardiol. 2007; 88(2):187-92. Portuguese.

12. Mion Júnior D, Pierin AM, Lessa I, Nobre F. Aparelhos, técnicas de medida da pressão arterial e critérios de hipertensão adotados por médicos brasileiros. Estudo exploratório. Arq Bras Cardiol. 2002; 79(6):593-6.

13. World Health Organization. Replacement of mercury thermometers and sphygmomanometer in health care. Technical guidance [Internet]. Geneva: WHO; 2011 [cited 2011 Out 10]. Available from: http://whqlibdoc.who.int/ publications/2011/9789241548182_eng.pdf.

14. O'Brien E, Petrie J, Littler W, de Swiet M, Padfield PL, O'Malley K, et al. The British Hypertension Society protocol for the evaluation of automated and semi-automated blood pressure measuring devices with special reference to ambulatory systems. J Hypertens. 1990; 8(7):607-19.

15. American National Standards Institute. American National Standard for Eletronic or Automated Sphygmomanometers. Arlington(VA): Association for the Advancement of Medical Instrumentation; 1987.

16. O’ Brien E, Waeber B, Parati G, Staessen J, Myers MG. Blood Pressure measuring devices: recommendations of the European Society of Hypertension. BMJ. 2001;322(7285):531-6.

17. Mion Júnior D, Pierin AM, Alavarce DC, Vasconcelos JH. The results of the campaign for evaluating sphygmomanometers accuracy and their physical conditions. Arq Bras Cardiol. 2000;74(1):31-8.

18. Turner MJ, Irwig L, Bune AJ, Kam PC, Baker AB. Lack of sphygmomanometer calibration causes over - and underdetection of hypertension: a computer simulation study. J Hypertens. 2006;24(10): 1931-8.

19. Arcuri EA, Rosa SC, Scanavini RM, Denzin GS. Arm and forearm blood pressure measurements as a function of cuff width. Acta Paul Enferm. 2009;22(1):37-42.

20. Pierin AM, Alavarce DC, Gusmão JL, Halpern A, Mion D Jr. Blood pressure measurement in obese patients: comparison between upper arm and forearm measurements. Blood Press Monit. 2004, 9(3):101-5.

21. Rabello CC, Pierin AM, Mion Júnior D. [The healthcare area professionals' knowledge of blood pressure measurement]. Rev Esc Enferm USP. 2004; 38(2):127-34. Portuguese. 